\title{
Turning Dynamics and Passive Damping in Flapping Flight
}

\author{
B. Cheng ${ }^{1}$, S.N. Fry ${ }^{2}$, Q. Huang ${ }^{1}$, W.B. Dickson ${ }^{3}$, M.H. Dickinson ${ }^{3}$ and X. Deng ${ }^{1 *}$
}

\begin{abstract}
-we investigated whether flapping flight has an inherent stability by analyzing the inertial and aerodynamic effects of flapping wings on body dynamics. Based on wing and body kinematics of free flying fruit flies during rapid maneuvers, we found a passive counter torque due to body rotation. It is indentified both in simulation through quasi-steady state aerodynamic model and through experiments on a dynamically scaled robotic wing. An analytical form is derived correspondingly. In the turning yaw axis, the estimated damping coefficient of flapping wings is significantly higher than body frictional damping; this indicates a passive deceleration during turning. By simulating insect to rotate about each principal axis of inertial and body frames, we calculated the corresponding damping coefficients, and further analyzed the attitude stability. The result reveals that, passive damping of flapping flight, while does not necessarily lead to a stable full body dynamics, provides a considerable passive restoring torque that could be critical for flight stabilization and control in the design of micro aerial vehicles. Preliminary analysis on the scaling parameters of passive damping was also performed.
\end{abstract}

\section{INTRODUCTION}

$\mathrm{F}$ lapping flight mechanism adopted by flying insects and birds, imparts unprecedented maneuverability over conventional aircrafts. Directly related to maneuverability, stability of flapping flight is of great interest. It was found both in tethered insect experiments and computational studies that, flapping flight is inherently unstable in longitudinal dynamics[1, 2]. However, insects are capable of maintaining a fixed body orientation under the disturbance of environment. It is observed in real insect flight that, the angular position of insect is held constant during cruising flight and abruptly change to a new direction by rapid turns, known as saccades[3]. Thus, the question of how the insect manage to stabilize itself while possessing such a great maneuverability is of great importance both for biological analysis and micro aerial vehicles (MAVs) design.

During saccade, as insect actively generate a large yaw torque to start turning, the mechanism for deceleration is still undetermined. While previous models assumed body friction play a major role to stop turning, S.Fry et al argued that inertia dominates the tuning dynamics[4], and active counter torque is required. However, both sides didn't consider the effect of body angular velocity during the deceleration. In this study,

Manuscript received September 15, 2008. This work was supported in part by NSF Grant\#0545931. ' Department of Mechanical Engineering, University of Delaware, 126 Spencer Laboratory, Newark, DE 19716, Email: deng@udel.edu; ${ }^{2}$ Institute of Neuroinformatics, ETH/University of Zurich, Switzerland; ${ }^{3}$ Department of Bioengineering, California Institute of Technology, Pasadena, CA. we systematically investigate the effect of body rotation on the aerodynamic force and torque production during saccade of fruitfly Drosophila, and we seek a comprehensive investigation of the mechanism of passive stability in flapping flight.

\section{MATERIAL AND METHODS}

\section{A. Analysis on simplified kinematics}

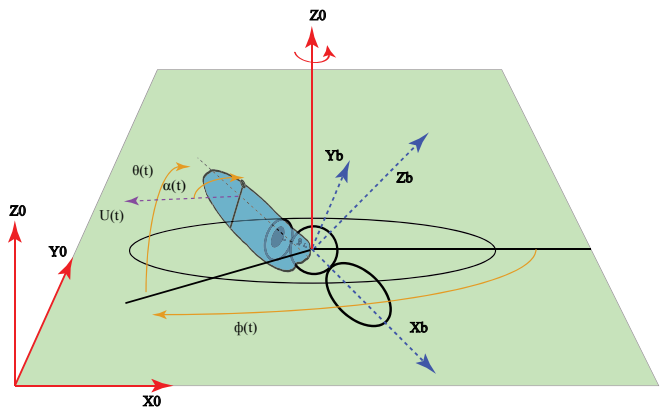

Fig. 1. Simulation model: head, thorax and abdomen of the fruitfly are modeled as ellipsoids. See Appendix (Table 2) for morphological data. Axis systems: body frame $\left(x_{b}, y_{b}\right.$ and $\left.z_{b}\right)$; inertial frame $\left(x_{0}, y_{0}\right.$ and $\left.z_{0}\right)$. Wing kinematics are specified by three Euler angles stroke angle $(\phi)$, deviation angle $(\theta)$ and rotation angle $(\varphi)$. Angle of attack $(\alpha)$ is defined as the angle of wing chord and tangential of wing's trajectory. And unless otherwise mentioned, this paper follows the conventions and nomenclature of [5].

To investigate the effect of body rotation on aerodynamic torque production during saccade, we simulated the rotation and calculated the resultant torque based on a quasi-steady aerodynamic model[6]. The coordinate system and kinematics parameters used throughout this paper are summarized in Fig. 1. An earth fixed inertial coordinate frame $x_{0}, y_{0}, z_{0}$ and a body-centered coordinate frame $x_{b}, y_{b}, z_{b}$ are introduced to give the reference frames for wing and body kinematics of the fly. The stroke plane is oriented with a fixed pitch angle (free body angle $\chi_{0}$ ) from the body frame, and the two frames share a same origin located at the center of mass. In this simulation the stroke plane is horizontal and its coordinates are aligned with the inertial frame. Three Euler angles, termed as stroke angle $(\phi)$, deviation angle $(\theta)$ and rotation angle $(\varphi)$ are employed to describe the wing kinematics. For simplifications, the head, thorax and abdomen of the fruit fly are modeled as ellipsoids (Fig. 1). The mass and inertia matrix are calculated based on the measured morphological data (Appendix C, Table 2). The insect was simulated to rotate with each principal axis of inertial frame and body frame with specified wing flapping kinematics (Fig. 2), and the aerodynamic forces on the wing were calculated based on the quasi-steady aerodynamic model[6]. We calculated the aerodynamic torques on the body frame assuming center of pressure located at $70 \%$ along the wing span $[7,8]$. 
First, we used a wingbeat frequency of $212 \mathrm{~Hz}$ and wing kinematics described in Fig. 2AB, both mimicking those of the fruit fly Drosophila during free flight. We simulated the flight with a inertial frame yaw turning velocity of $1907.5 \mathrm{degs} / \mathrm{s}$ which is close to the average yaw angular velocity of a fruitfly during saccade (Fig. 8C) in measurements [4]. We then calculated the resultant inertial frame yaw torque, and denoted it as passive counter torque. As a comparison, we simulated the flight with the same body angular velocity with both wings stationary at a constant angle of attack of 90 degrees (Fig. 2C). Without wing flapping, the wings and body comprise a single rigid object and the resultant counter torque is induced solely by the turning velocity. Because wing flapping affects the magnitude and direction of instantaneous forces and torques[9][10], the measured resultant torque is expected to differ significantly under these two conditions. We then calculated the damping coefficient for yaw rotation based on the measured counter torque for both cases.

The above simulations were then repeated for the remaining principal axes in both the inertial and body frames $\left(x_{0}, y_{0}, z_{0}, x_{b}, y_{b}\right.$ and $\left.z_{b}\right)$ (Fig. 2D), and the corresponding counter torques were calculated. For roll and pitch, the angular velocity was also selected to be close to their mean value observed in free flight insect data (Fig. 8C), Table I lists the turning axis and corresponding velocities. Here $\omega$ represents the body angular velocities represented in either inertial $(0)$ or body frame $(b)$. For each turning axis, we calculated the damping coefficients based on the averaged torques. We then systematically varied the flapping frequency and turning velocity to investigate how they affected the stroke averaged counter torque.

TABLE I

Turning axes and corresponding angular velocities

\begin{tabular}{ccccccc}
\hline \hline Turning Axis & $z_{0}$ & $z_{b}$ & $y_{0}$ & $y_{b}$ & $x_{0}$ & $x_{b}$ \\
\hline Angular velocity $\omega$ & 1.91 & 1.91 & 0.933 & 0.933 & 4.63 & 4.63
\end{tabular}

Angular velocity has a dimension of $10^{3} \mathrm{degs} / \mathrm{s}$. Values of the turning velocity are selected near the maximum turning velocity of real saccade of fruit fly.

\section{B. Analysis with real insect data}

Using a three high-speed video camera system, Fry et al. captured the wing and body kinematics of fruit fly Drosophila melanogaster in free flight, and measured the aerodynamic forces by playing the wing kinematics in body-centered frame (Fig. 3A) on a dynamically scaled robotic model [4]. Here, we based our simulation and experiments on the same flight data, and superimposed (through coordinate transformation) the body angular velocities onto the wing kinematics. We played the modified wing kinematics (Fig. 3B) on the robotic wing and evaluated the influence of the body motion on the aerodynamics force and torque production. Fig. 3 shows the original (Fig. 3A) and modified (Fig. 3B) wing kinematics of fruit fly saccade data in this study.
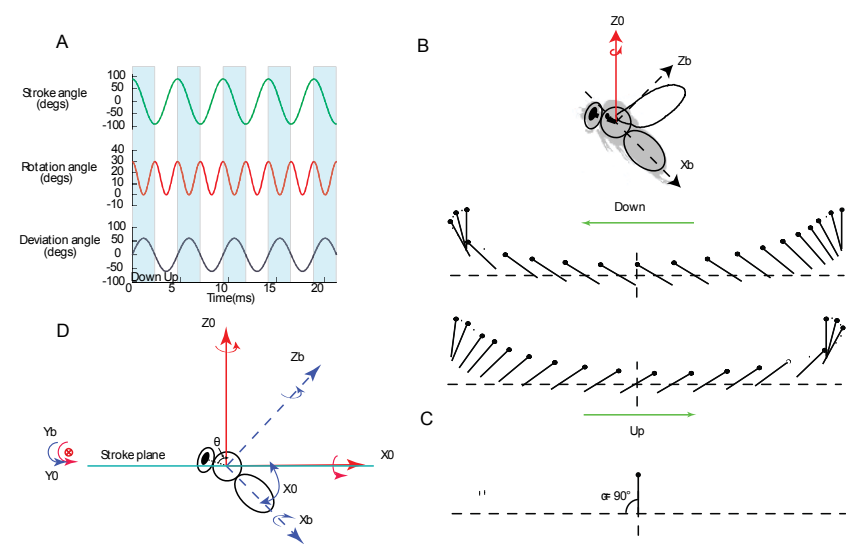

Fig. 2. (A) Wing kinematics, plotted over 4.5 flapping cycles (total simulation is 10 flapping cycles). Blue and white backgrounds mark down and up strokes. Stroke angle, (green), deviation angle and rotation angle ([for definitions see [5]]), vary as sinusoids $(\pi / 2) \cos \omega t,(\pi / 12) \cos 2 \omega t+\pi / 6$ and $(\pi / 3) \sin \omega t$ respectively, which approximates the wing kinematics of freely hovering Drosophila (B) Wing motion with the kinematics, black line denotes the wing chord, with filled cycle marking leading edge. (C) Wing motion with the wing fixed at body frame at the middle of the stroke, and sweeping through the air at angle of attack 90 degrees. (D) Six turning axes in the simulation, consisting three principal axes of inertial frame $\left(x_{0}, y_{0}, z_{0}\right)$ denoted as inertial roll, pitch and yaw and of body frame $\left(x_{b}, y_{b}, z_{b}\right)$ denoted as body roll, pitch and yaw, note that $y_{0}$ and $y_{b}$ coincide with each other. Free body angle $\chi_{0}$ is set to be 45 degs.

\section{Experiment setup}

We measure aerodynamic forces on flapping wings using a dynamically-scaled mechanical flapper, a modified version of the one described in Dickinson et al [10] and Sane and Dickinson [11]. A detailed description is given by[12]. In the current experiment, the instantaneous force and torque acting on the wing were measured using a six-component force-torque sensor (ATI NANO-17, Apex, NC) attached to the wing holder. Wings are made of PET-G0.06 with the shape of a fruit fly wing and a length of $18 \mathrm{~cm}$. The wing base is attached to a holder affixed on the gear box. The wing along with the gearbox $(2.54 \mathrm{~cm} \mathrm{X} 2.54 \mathrm{~cm} \mathrm{X} 2.54 \mathrm{~cm})$ was immersed in a tank (46 cm width X $41 \mathrm{~cm} \mathrm{X} 152 \mathrm{~cm}$ length) filled with mineral oil (Kinematic viscosity $=20 \mathrm{cSt}$ at $20^{\circ} \mathrm{C}$, density $=850 \mathrm{~kg} / \mathrm{m}^{3}$ ). This overall set-up enabled us to move the wings along pre-determined insect-like kinematic patterns while simultaneously measuring the forces on the wings. The Reynolds number for our experiments was calculated at 250 using the equation:

$$
\overline{R e}=\frac{4 \theta R^{2} n}{v(A R)}
$$

where $A R$ is the aspect ratio, $\mathrm{n}$ is the wing beat frequency, $R$ is wing length, $\theta$ is the wing beat amplitude( peak-to-peak in radians) and $v$ is the kinematic viscosity of the fluid [13]. Although the Reynolds number for Drosophila at hovering is around $150[4,7]$, which is lower than the one in our experiment, we expect this difference to have negligible effects because the viscous force does not dominate flight at these Reynolds numbers as confirmed by both CFD simulation and flapper experiments[14]. 


\section{Aerodynamic torque measurement and calculation}

Measurements were performed with a single robotic wing because wing-wing interactions are negligible during hovering free flight[7]. Forces and torques measured on the wing are scaled versions of those on the real fly, according to the force scaling rule (Appendix D). The body frame torques were obtained through coordinate transformation. If the insect body is modeled as a rigid body then its complete dynamics is described by the Newton-Euler equations of motion (Eqn. 1):

$\left[\begin{array}{cc}m I & 0 \\ 0 & \mathfrak{T}\end{array}\right]\left[\begin{array}{c}\dot{v}^{b} \\ \dot{\omega}^{b}\end{array}\right]\left[\begin{array}{c}\omega^{b} \times m v^{b} \\ \omega^{b} \times \mathfrak{T} \omega^{b}\end{array}\right]=F^{b}=\left[\begin{array}{c}f_{a} \\ \tau_{a}\end{array}\right]+\left[\begin{array}{c}f_{f} \\ \tau_{f}\end{array}\right]+\left[\begin{array}{c}f_{g} \\ 0\end{array}\right]$

where $m$ is the body mass, $I$ is the identity matrix, $\mathfrak{I}$ is the body moment of inertia matrix, $\omega^{b}$ and $v^{b}$ are the instantaneous body frame angular and translational velocities. The terms $f_{a}$ and $\tau_{a}$ represent the aerodynamic forces and torques; $f_{f}$ and $\tau_{f}$ are the frictional damping forces and torques acting on the fly's body, and $f_{g}$ is the body gravitational force. Gravitational force of the wing is much smaller than the other components and is thus ignored. The quantities on the left hand side of the equation were calculated based on the body kinematics and morphology data described in Appendix C. Theoretically, the time trace of the estimated quantities on the left side (predicted force and torque) and those measured on the right side of the equation should match with bounded discrepancies, which arise from measurement errors and uncertainties in the morphological parameters.
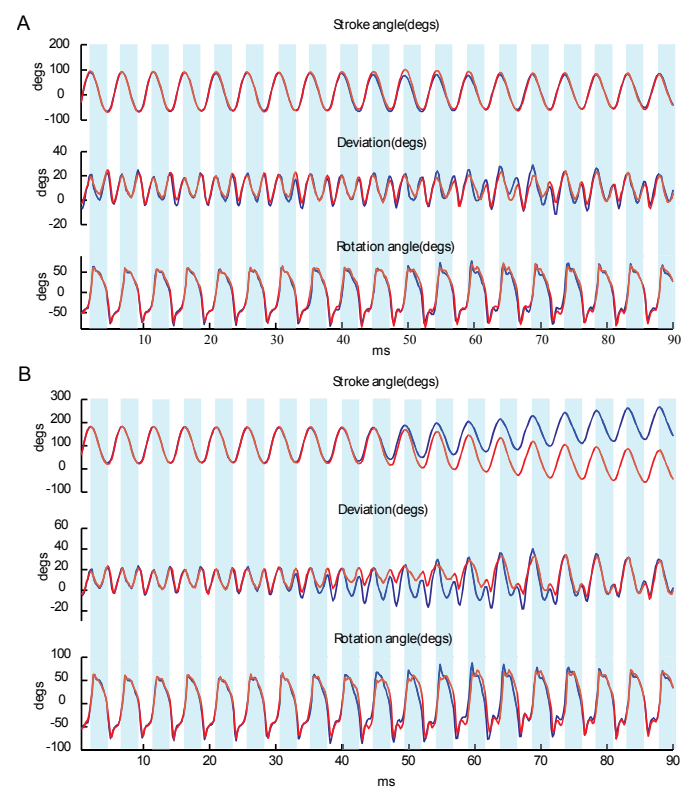

Fig. 3. (A) Wing kinematics (stroke angle $\phi$, deviation angle $\theta$ and rotation angle $\varphi$, in body-centered frame of the left (blue) and right (red) wing re-plotted from [4]. (B) Absolute wing kinematics (right and left wings are shown in red and blue, respectively) which take into considerations the body rotation. All angles are derived from ZYX Euler angles for coordinate transformation, for detail please refer to[15].

\section{E. Inertia analysis in flapping wing}

To rule out the possibility of inertia induced stability (similar mechanism as gyroscopic effect); we investigated the effect of wing inertia force on body dynamics, using a custom designed dynamic model. Note that although insect wing pair only has a small portion of the total insect weight, under high flapping frequency, its inertia force could still be significant.

Body 1 is a rod with negligible length of radius. It is connected to the fixed inertial frame by a frictionless global joint, and its position is described by $\theta$ and $\phi$. Another two rods (negligible radius and mass, denoted as Body 2 and 3), both of which has a mass point attached to one end, are connected to Body 1 by two frictionless global joints at point A. Body 2 and 3 are flapping symmetrically with $180^{\circ}$ stroke amplitude at the stroke plane perpendicular to the Body1 (Axis $Z_{b}$ ), their positions are specified by a single parameter $\psi$. They are driven by two weightless motors mounted on Body 1. A complete dynamics of Body 1, 2, 3, three frictionless joints and two motors were established under gravity. Given the initial positions and velocities of $\theta$ and $\phi$, the dynamics was simulated under a prescribed flapping frequency of Body 2 and 3. If we change Body 2 and 3 to a rotating disc, this model will become a gyroscope, and a gyroscopic effect is expected.

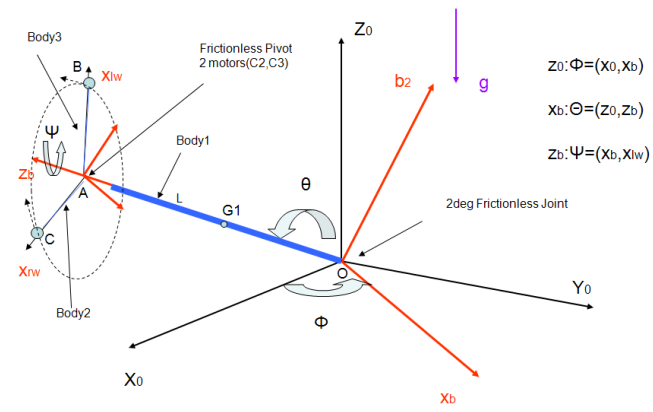

Fig. 4 Schematic plot the custom designed dynamic model. Three axis systems attached to each object are introduced: Body 1: $\left(x_{b}, y_{b}\right.$ and $\left.z_{b}\right)$, Body 2: $\left(x_{l w}, y_{l w}\right.$ and $\left.z_{r w}\right)$, Body $3:\left(x_{r w}, y_{r w}\right.$ and $\left.z_{r w}\right)$.

\section{RESULTS}

\section{A. Counter torque induced damping during turning}

We first simulated rotation around the inertial yaw axis and calculated the resultant counter torque with rigid wings at an angle of attack of 90 degrees at $2.37 \times 10^{-11} \mathrm{~N} \mathrm{~m}$. With the wings flapping symmetrically (Fig. 2) under otherwise identical conditions, the counter torque averaged over 10 stroke cycles is $9.54 \times 10^{-10} \mathrm{~N} \mathrm{~m}$, or about 40 -fold increased compared to the non-flapping case (Fig. 5A). It is clear to see that counter torque was greatly enhanced by the flapping of the wing. The magnitude of this counter torque is about half of the maximum body frame yaw torque of $2 \times 10^{-9} \mathrm{~N} \mathrm{~m}$ resulting from active turning using asymmetric wing motion [4], and is very close to the total body frame yaw torque of $1 \times 10^{-9} \mathrm{~N}$ m predicted by the Newton-Euler equation based on measured body saccade kinematics (Fig. 8A). Our result confirms that passive counter torque is at least partially responsible for the deceleration during saccades, and that the fruit fly may only need to 
produce a small additional amount of active counter torque to completely terminate the body rotation.

The existence of body velocities induced counter torque is clear to justify with a few simplifying assumptions. Most of the aerodynamics force on the wing comes from delayed stall effect, which gives a translational force proportional to the square of wing velocity. If we assume that the moment arms (defined as the vector from the center of mass of the body to the center of pressure on the wing) for both wings are the same, we can uniformly write the instantaneous yaw torques in the stroke plane during up and down stroke as (Appendix B2)

$$
\tau_{\text {total }}=-C_{N}(t) \rho R^{4} \bar{c} \hat{r}_{2}^{2}(S) \hat{r}_{c p} \Phi n \cos (\varphi)\left(\frac{d \widehat{\phi}}{d \hat{t}}\right) \omega_{b}
$$

where $\rho$ is air density; $R$ is the wing length; $C_{N}(t)$ reprensents the instantaneous wing force coefficient due to delayed stall (function of angle of attack $\alpha(t)$ ); $\omega_{b}$ is the instantaneous wing angular velocity; $\varphi$ is the rotation angle, for simplicity we have assumed $\varphi$ is symmetric for left and right wings; $(d \hat{\phi}) /(d \hat{t})$ is the normalized wing angular velocity; $\Phi$ and $n$ are the wing flapping amplitude and frequency; $\hat{r}_{2}^{2}(S)$ and $\hat{r}_{c p}$ are second moment of wing mass and normalized center of pressure on the wing.
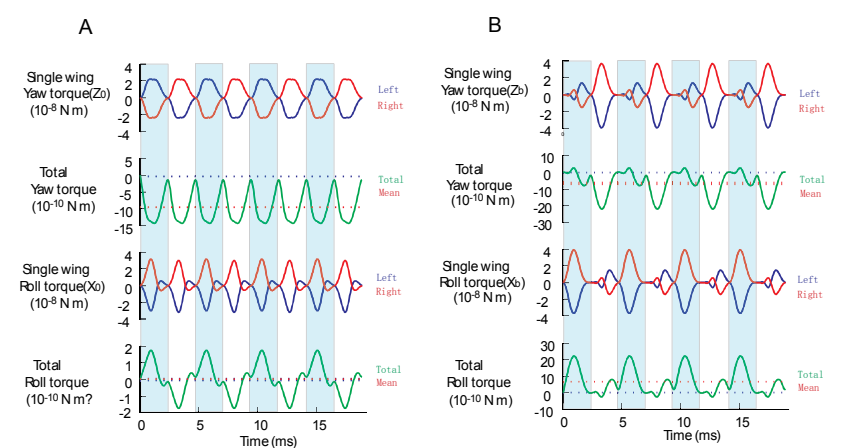

Fig. 5. Simulated wing torques (A) Inertial frame yaw and roll torques for Left (blue), right (red) wing. Total yaw and roll torques are plotted separately (green), and with its mean value(dotted red) and values for no wing flapping case(dotted blue) (B) Body frame yaw and roll torques for Left (blue), right (red) wing.

Since it is reasonable to view passive counter torque as a form of aerodynamic damping due to its linear relationship with body angular velocity, we evaluated the mean damping coefficients. Here we define the corresponding aerodynamic damping coefficient as:

$$
C_{i-j}=\frac{\tau_{i-j}}{\omega_{i-j}}
$$

Damping coefficients in different frames of reference can be obtained through the torques and velocities in that frame by coordinate transformations. For example, both the body frame yaw torque $\left(\tau_{z b-z 0}\right)$ and velocity $\left(\omega_{z b-z 0}\right)$ for the inertial frame yaw axis can be obtained from coordinate transformation and results in $6.75 \times 10^{-10} \mathrm{~N} \mathrm{~m} \mathrm{~s}$ and $1.35 \times 10^{3} \mathrm{degs} / \mathrm{s}$ respectively, (instantaneous and mean values are shown in Fig. 5B). The corresponding damping coefficient $C_{z b-z 0}$ for the body yaw axis is $28.69 \times$ $10^{-12} N \mathrm{~ms}$, which is the same as $C_{z 0-z 0}$. As will be shown later in this section, this equality only holds true for certain cases of turning.

To compare the role of inertia and damping in flight dynamics during turning, we investigate the dynamics in the body coordinate frame. We separate the total yaw torque into an active component, which is due to asymmetric wing motion and a passive component due to body rotation. Here we neglect the cross product terms in Eqn. 1 since the magnitude of the cross term is near $1 / 30$ of the value of the other terms calculated based on free flight data of a saccade [4] and only examine the body frame yaw dynamics, which simplifies to (Appendix A):

$I_{\text {yaw }} \dot{\omega}_{\text {yaw }}^{b}=\tau_{\text {active_yaw }}-C_{z b-z 0} \omega_{z b-z 0}-C_{\text {friction }} \omega_{z b-z 0}$

where $\tau_{\text {active_yaw }}$ is the active component of yaw torque, $\tau_{\text {counter_yaw }}$ is the passive yaw counter torque $(-6.75 \times$ $\left.10^{-10} \mathrm{~N} \mathrm{~m}\right), I_{\text {yaw }}$ is the yaw moment of inertia estimated based on body morphologies (Appendix C, 4.971 $\times$ $\left.10^{-12} \mathrm{~N} \mathrm{~m} \mathrm{~s}^{2}\right), \omega_{z b-z 0}$ is the angular velocity about the body yaw axis $\left(1.35 \times 10^{3} \mathrm{degs} / \mathrm{s}\right)$, and $C_{\text {friction }}$ is the frictional damping coefficient of the body. The total damping coefficient, $C=C_{z b-z 0}+C_{\text {friction }}$ was estimated as $29.21 \times$ $10^{-12} N \mathrm{~ms}$ in the first set of simulations with symmetric wing motion (Fig. 2AB). The body frictional component, $C_{\text {friction }}$, was estimated by Stokes' law $\left(0.52 \times 10^{-12} \mathrm{~N} \mathrm{~m}\right.$, [4]), whereas $C_{z b-z 0}$ was calculated as $28.69 \times 10^{-12} \mathrm{~N} \mathrm{~ms}$, or roughly 56 times the value of body frictional damping.

To get a comprehensive view of damping coefficient in all three orthogonal axes, and further analyze the dynamic stability of flapping flight, we simulated the situation which insect is turning separately about $x_{0}, y_{0}, z_{0}, x_{b}, y_{b}$ and $z_{b}$ axes (Fig. 2D) with a symmetric wing stroke pattern (Fig. 2AB). The results show similar counter torques in both roll and pitch direction as documented in Table II. It is apparent that, for the rotations round the principal axes of inertial frame, damping is most prominent about the turning axis upon which the angular velocity is specified (values of counter torques on other axes are negligible). In other words, the resultant torque is roughly collinear with the turning axis. Significantly, this is also true for rotations about $x_{b}$ and $z_{b}$, which have a fixed orientation in the inertial frame coordinates. Thus, it is highly suspicious that such effect holds for any rotation axes, which has not been systematically addressed in current study.
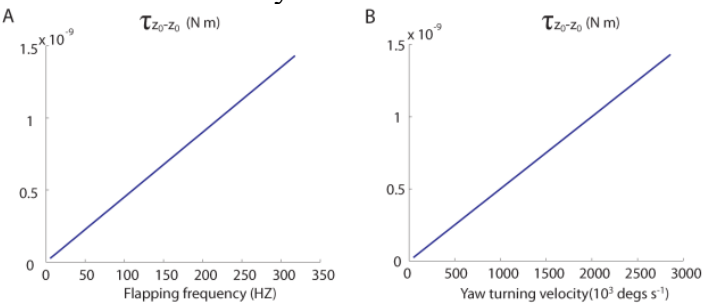

Fig. 6. (A) Counter torque by varying the flapping frequency. (B) Counter torque by varying the turning velocity of inertial yaw. 
The counter torque is a part of the total aerodynamic torque which is an inherently nonlinear function of body velocity and wing kinematics. We systematically varied the flapping frequency and turning velocity to quantify their effects on passive counter torque production and tested for its linearity, as predicted by Eqn. 2. Unsurprisingly, the result confirms their linear relationship (Fig. 6), and the corresponding damping coefficients is a constant.

TABLE II

Toques and damping coefficients

\begin{tabular}{l|cccccc}
\hline \hline $\begin{array}{l}\text { Turning } \\
\text { Axis }\end{array}$ & $z_{0}$ & $z_{b}$ & $y_{0}$ & $y_{b}$ & $x_{0}$ & $x_{b}$ \\
\hline Angular & 1.91 & 1.91 & 0.933 & 0.933 & 2.0 & 2.0 \\
velocity & & & & & & \\
\hline$\tau_{z b-}$ & $/$ & -9.48 & $/$ & 0 & $/$ & 0.06 \\
$\tau_{y b-}$ & $/$ & 0.03 & $/$ & -2.19 & $/$ & -0.03 \\
$\tau_{x b-}$ & $/$ & 0.06 & $/$ & 0 & $/$ & -9.95 \\
$\tau_{z 0-}$ & -9.54 & & 0 & & 0 & \\
$\tau_{y 0-}$ & 0 & & -2.19 & & 0 & \\
$\tau_{x 0-}$ & 0 & & 0 & & -9.88 & \\
$C_{z b-}$ & $/$ & 28.45 & $/$ & 0 & $/$ & -0.17 \\
$C_{y b-}$ & $/$ & -0.09 & $/$ & 13.46 & $/$ & 0.09 \\
$C_{x b-}$ & $/$ & -0.18 & $/$ & 0 & $/$ & 28.50 \\
$C_{z 0-}$ & 28.69 & $/$ & 0 & $/$ & 0 & $/$ \\
$C_{y 0-}$ & 0 & $/$ & 13.46 & $/$ & 0 & $/$ \\
$C_{x 0-}$ & 0 & $/$ & 0 & $/$ & 28.32 & $/$ \\
\hline \hline
\end{tabular}

Dimension of angular velocities is $10^{3} \mathrm{degs} / \mathrm{s}$ Dimension of torques is $10^{-10} \mathrm{~N}$ mand dimensions of damping coefficients is $10^{-12} \mathrm{~N} \mathrm{~m} \mathrm{~s}$. Note that negative torque corresponds to positive damping coefficient.

Lastly, the passive stability due to passive counter torque was examined by looking at the system matrix A for the averaged attitude dynamics, linearized at hovering equilibrium (detail derivation is not presented here, for a similar derivation of longitudinal dynamics refer to[2]):

$\left[\begin{array}{c}\delta \dot{p} \\ \delta \dot{q} \\ \delta \dot{r} \\ \delta \dot{\phi} \\ \delta \dot{\theta} \\ \delta \dot{\psi}\end{array}\right]=\left[\begin{array}{cccccc}-248.91 & -0.79 & 1.48 & 0 & 0 & 0 \\ 0 & -26.60 & 0 & 0 & 0 & 0 \\ 0.36 & 0.18 & -57.23 & 0 & 0 & 0 \\ 1 & 0 & 1 & 0 & 0 & 0 \\ 0 & 1 & 0 & 0 & 0 & 0 \\ 0 & 0 & 1.4142 & 0 & 0 & 0\end{array}\right]\left[\begin{array}{c}\delta p \\ \delta q \\ \delta r \\ \delta \phi \\ \delta \theta \\ \delta \psi\end{array}\right]$

where angular velocity $\omega_{b}=[p, q, r]^{T}$, and the angular position of the insect body is described by three Euler angles (roll, $\phi$; pitch, $\theta$ and yaw $\psi$ ). The eigenvalues of A are: -57.2 , $-248.9,-26.6,0,0,0$. The zero eigenvalues indicate that the linearized system is marginally stable, and the stability of the corresponding nonlinear system cannot be determined based on the current linearized model. Thus, further analysis is required to determine the passive stability of attitude dynamics. Furthermore, the overall stability of insect flight involves a full body dynamics including attitude and translational components. Previous studies have identified unstable modes in the hovering longitudinal dynamics based on CFD simulations [16] and tethered flight experiments[2]. On the other hand, despite the failure of the linearized model in determining the passive attitude stability, passive counter torque does serve as a stabilizing factor for flapping flight as it make harder for insect to maneuver and increases the damping of flapping flight .

\section{B. Wing inertia analysis}

First, we simulated the dynamic model with a rotating disc in replacement of the flapping wings. As predicted by gyroscopic effect, Body 1 did not fall down but was oscillating about $x_{b}$ axis (Fig. 7, indicated by the oscillation of $\theta$ ). Concurrently, it's rotating about vertical axis $z_{0}$ at near constant angular velocity ( $\dot{\phi} \approx$ constant).For simulation with flapping wings, we didn't observe a similar result as in the first case, while given various initial conditions and flapping frequencies. When simulation started, Body 1 simply felt down and kept oscillating (Fig. 7 oscillation of $\phi$ ) like a pendulum.

Thus, the result shows that the passive stability due to wing inertia doesn't exist and won't contribute to the total stability of flapping flight.
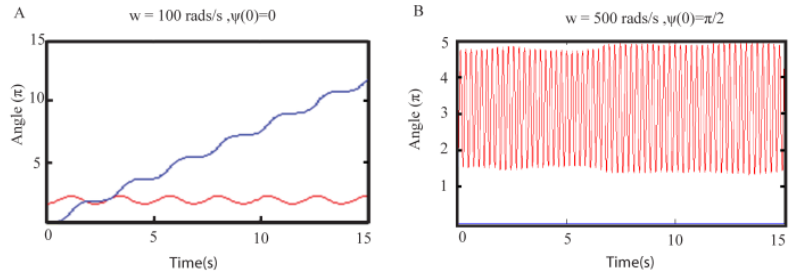

Fig. 7. Instantaneous value of $\phi$ (blue) and $\theta$ (red) for (A) gyroscope (B) flapping wings. As indicated by the time trace of $\theta$ that Body 1 is oscillating like a pendulum for a flapping wing pair.

\section{Scaling of counter torque and passive stability}

In order to analyze the scaling of the passive counter torque, we analytically write the averaged body acceleration of insect due to counter torque as:

$$
\begin{aligned}
\dot{\omega}_{p} & =-\omega \frac{{\overline{C_{N}}}_{\bar{l}} \rho R^{4} \overline{\mathrm{c}}_{2}^{2}(\mathrm{~S}) \hat{\mathrm{r}}_{\mathrm{cp}} \Phi n \overline{(d \widehat{\phi} / d \hat{t})}}{m(a R)^{2}} \\
& =-\mathrm{C}_{m} \frac{4 \mathrm{~g} \omega \hat{\mathrm{r}}_{\mathrm{cp}}}{a^{2} \Phi n \overline{(d \hat{\phi} / d \hat{t}) R}} \propto-\frac{\omega}{\Phi n R}
\end{aligned}
$$

where $C_{m}=\frac{\overline{C_{N}} \rho \Phi^{2} n^{2} R^{3} \bar{c}_{2}^{2}(\mathrm{~S}) \overline{(d \hat{\phi} / d \hat{t})}^{2}}{4 m g}, m$ is the insect body mass, $a$ is a lumped parameter of insect body yaw MOI, and other parameters follow the same conventions described in previous sections. Since $C_{m}$ is a ratio for body weight and mean lift force, its value is expected to be a rough constant for different scale of insects. Thus, as demonstrated in the Eqn. 6, the passive acceleration is inverse proportional to wing length $(R)$, flapping amplitude $(\Phi)$ and frequency $(n)$. Preliminary analysis based on insect wing kinematics and morphological data, shows that $\dot{\omega}_{p}$ for fruitfly Drosophila (higher flapping frequency but much smaller wing length) is $4 \mathrm{x}$ than the humming bird. As observed in the free flight of the above two species, when decelerating from a same maximum angular velocity, humming bird needs roughly $4 \mathrm{x}$ greater time to reach a half of the initial velocity, indicating an agreement with our predication. Therefore, a proper selection of 
$\Phi, n$ and $R$ is critical for the MAV flight performance and control in terms of passive stability.

\section{Torque production of saccade}

To assess the effects of passive counter torque under realistic free-flight conditions, we compared the body frame yaw torque generated by two sets of wing kinematics in presence and absence of body rotation (Fig. 3). Results are presented in Fig. 8. The substantial difference between the two confirms our previous analysis that body angular velocity plays an important role in turning dynamics while passive counter torque cannot be neglected. During saccades, flies not only produce high angular velocities about the yaw axis, but also about the roll and pitch axes (Fig. 8C), and the combined total angular velocity influences the aerodynamic forces on the wings and causes the discrepancy between the torque production of these two cases.

We divided the time trace in the figure into four phases (Fig. 8) based on hovering, acceleration, constant body yaw velocity and deceleration (red line in Fig. 8C). In phase I during which the fly is almost hovering in place, it has a near zero yaw angular velocity. However, there exists considerable amount of angular velocity about the roll and pitch axes (Fig. 8C). During this phase, the difference of yaw torque between the two cases is small and may be due to coupling with the non-zero pitch and roll angular velocities.

At the onset of the saccade (Phase II), the fly accelerates and a large active yaw torque in body frame is generated by active wing motion (red line in Fig. 8A). Meanwhile, the total yaw torque is smaller than the active yaw torque because the increasing yaw angular velocity leads to strong counter torque (blue curve in Fig. 8A). During this period, the instantaneous turning axis roughly corresponds to the body yaw axis. Because the turning axis does not coincide with the vertical axis in the inertial frame (the body pitch angle is roughly $45^{\circ}$ inclined relative to the horizontal plane), torque about it will induce body roll (banking) and a pitch down motion as previously described for free flight $[4,17]$.

During phase III, yaw velocity reached its maximum and remained roughly constant (but not for all saccades, see[4]). Active yaw torque (Fig. 8A) is positive, corroborating the CFD results of [18], as the fly continues to produce turning torque by asymmetric wing motion. However, the total torque is reduced by passive counter torque. We also note that there exists a large oscillation about the roll axis at the flapping frequency with a maximum magnitude at about twice the yaw angular velocity (Fig. 8C). Despite the possibility of measurement amplification of roll angular velocity, this might provide evidence for active modulation of wing kinematics because passive damping could not lead to such an oscillation.

During phase $I V$, the large oscillation about the roll axis decreases and the fly is decelerating (Fig. 8C). Note that the fly roughly takes two wing beats to decelerate to half the maximum angular velocity and the entire deceleration lasts about $20 \mathrm{~ms}$. The time constant calculated based on counter torque passive damping is around $17 \mathrm{~ms}$, indicating that passive damping could be mostly responsible for the measured deceleration. However, during this stage the fly also appears to generate active decelerating torque as evidenced by its asymmetric wing kinematics [4]. This implies that although passive counter torque could serve as a main factor of deceleration, active decelerating cannot be excluded. The angular velocity is mostly about the inertial yaw axis during this final stage of turning. From Fig. 8A, the active yaw torque without body rotational effects (red trace) is almost positive during the saccade, except at the end of the saccade starting around $70 \mathrm{~ms}$, and is greater than the total yaw torque either measured or predicted by the Euler equation (by calculating the left side of Eqn. 2). Toward the end of the saccade, the fly could assume a roughly symmetric stroke pattern and let passive damping reduce its angular velocity, while producing a small amount of active braking torque with an asymmetric wing stroke.

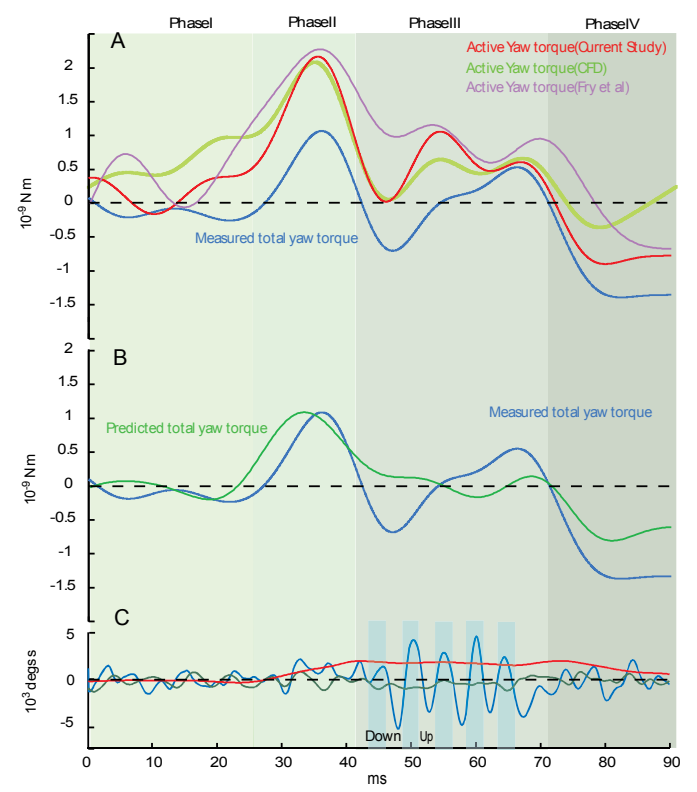

Fig. 8.(A) Measured body yaw torque with (Blue) and without (Red) body angular velocities superimposed on the wing kinematics, and their difference as the counter torque, CFD results (green[18]) and S. Fry's results (purple,[7]) without body velocities; (B) Left hand side of the Euler equation (green) compared with measured torque in our experiment (again blue). A strong positive turning torque and a negative restoring torque can be seen at the onset and end of the saccade. Because the cross terms in Euler equation is small compared to the other term, the estimated yaw torque is proportional to the yaw angular acceleration. (C) Body angular velocity about roll (blue), pitch (green) and yaw (red) axis.

\section{DISCUSSION AND FUTURE WORK}

In the flapping flight, for each perturbed turning axis, there always exists a restoring torque which functions as a passive damping(as indicated by the positive damping coefficients Table.2). However, instability could still exit in flapping flight because of the coupling. In the study by Taylor and Sun, they both indentified the unstable modes in longitudinal flight dynamics for flapping flight. As described in $[1,16]$, the calculated aerodynamics derivatives (similar to damping coefficients) show similar damping effects in roll, forward, 
and descending directions and serves as a stable factor for flapping flight (One can find similar damping in translational motion, i.e. forward and descending). Other cross terms, however, may cause the instability; for example, a disturbance of forward velocity during hovering will cause a large pitch moment that lead to an unstable longitudinal dynamics[1, 16]. Therefore, in flapping flight dynamics, the instability does exits due to coupling and need active stabilizations during hovering and maneuvering.

In this study, we focused our analysis of passive damping and stability on fruitfly Drosophila which is a relatively small scale fly. It is of great importance to investigate the scaling of body angular velocity induced passive damping on different scale of insects, and to use the results to help decide the design parameters of flapping wing MAV. We showed here the preliminary results that smaller scale fliers may has great passive stability as well as maneuverability over large scale fliers. Furthermore, in current analysis, an analytical form of passive counter torque for turning about yaw axis was derived; in the future work, we will seek to find similar analytic forms of counter torque for roll and pitch axis.

\section{APPENDIX}

(A)

If we neglect the cross product terms in the Euler equation, which are negligible compared to the inertia term, and only write terms in the yaw direction, Eqn. 1 may be simplified to:

$$
I_{\text {yaw }} \dot{\omega}_{\text {yaw }}^{b}=\tau_{\text {yaw }}
$$

In order to separate the different torque production mechanisms, we linearize the total yaw torque, $\tau_{\text {yaw }}$ with body yaw angular velocity $\omega_{\text {yaw }}^{b}$ at a reference point $\omega_{\text {yaw (ref) }}^{b}$. Thus, the total yaw torque is decomposed into an active torque produced by wing motion ( $\left.\tau_{\text {active_yaw }}\right)$, a passive counter torque due to body rotation $\left(\tau_{\text {counter_yaw }}\right)$, and a damping torque due to body friction $\left(\tau_{\text {body_fricition }}\right)$, as in Eqn. A2:

$$
\begin{aligned}
& \tau_{\text {yaw }}=\tau_{\text {yaw }}\left(\omega_{\text {yaw }}^{b}, \vec{\phi}_{l}, \frac{\partial \vec{\phi}_{l}}{\partial t}, \vec{\phi}_{r}, \frac{\partial \vec{\phi}_{r}}{\partial t}\right) \\
& =\tau_{\text {yaw }}\left(\omega_{\text {yaw }(\text { ref })}^{b}, \vec{\phi}_{l}, \frac{\partial \vec{\phi}_{l}}{\partial t}, \vec{\phi}_{r}, \frac{\partial \vec{\phi}_{r}}{\partial t}\right)+\left(\left.\frac{\partial \tau_{\text {yaw }}}{\partial \omega_{\text {yaw }}^{b}}\right|_{\omega_{\text {yaw }}^{b}=r e f}\right) \omega_{\text {yaw }}^{b} \\
& =\tau_{\text {active_yaw }}\left(\omega_{\text {yaw }(0)}^{b}, \vec{\phi}_{l}, \frac{\partial \bar{\phi}_{l}}{\partial t}, \vec{\phi}_{r}, \frac{\partial \bar{\phi}_{r}}{\partial t}\right)-\left(C_{z b-z 0}+C_{\text {friction }}\right) \omega_{\text {yaw }}^{b} \\
& =\tau_{\text {active_yaw }}-C_{z b-z 0} \omega_{z b-z 0}-C_{\text {friction }} \omega_{\text {zb-z0 }} \\
& =\tau_{\text {active_yaw }}-\tau_{\text {counter_yaw }}-\tau_{\text {body_friciton }}
\end{aligned}
$$

where $\vec{\phi}=(\varphi, \theta, \phi)$ and $\frac{\partial \vec{\phi}}{\partial t}$ represent the wing kinematics in body coordinates; $\omega_{\text {yaw }}^{b}\left(\omega_{z b-z 0}\right)$ is the body yaw angular velocity (defined in Eqn. 3 ); $C_{\text {friction }}$ is the body frictional damping coefficient; $C_{z b-z 0}$ is the damping coefficient due to passive counter torque (defined in Eqn. 3). Here we use negative signs in front of $C_{z b-z 0}$ and $C_{\text {friction }}$ because the damping opposes turning. We also use the fact that both passive damping and body frictional torque are linearly proportional to the body angular velocity. Note this separation is for better understanding of each mechanism and is not the true representation of torque production. In reality, force and torque are nonlinear functions of body velocity (translational and angular) and wing kinematics.

\section{(B)}

Here we provide a mathematical derivation of passive counter torque based on a quasi-steady analysis of instantaneous torque that takes into consideration of the effect of body rotation. The instantaneous left wing torque during upstroke is calculated as:

$$
\tau_{l-u p}(t)=F_{l-u p} R \hat{r}_{c p}=-\frac{1}{2} C_{N}(t) \rho R \bar{c}\left|\vec{U}_{w}+\vec{U}_{b}\right|^{2} R \hat{r}_{c p} \cos (\varphi)
$$

If there is no wing deviation, and denote:

$U_{w}=\left|\vec{U}_{w}\right|$ and $U_{b}=\left|\vec{U}_{b}\right|$ where $U_{w}=\left(\frac{\Phi n}{2}\right)\left(\frac{d \widehat{\phi}}{d \hat{t}}\right) R r_{2}(S)$ and $U_{b}=\omega_{b} R r_{2}(S)$, the equation becomes:

$\tau_{l-u p}(t)=-\frac{1}{2} C_{N}(t) \rho R^{4} \bar{c} \hat{r}_{2}^{2}(S) \hat{r}_{c p}\left(\left(\frac{\Phi n}{2}\right)\left(\frac{d \widehat{\phi}}{d \hat{t}}\right)+\omega_{b}\right)^{2} \cos (\varphi)$

Similarly:

$\tau_{l-\text { down }}(t)=\frac{1}{2} C_{N}(t) \rho R^{4} \bar{c}_{2}^{2}(S) \hat{r}_{c p}\left(\left(\frac{\Phi n}{2}\right)\left(\frac{d \widehat{\phi}}{d \hat{t}}\right)-\omega_{b}\right)^{2} \cos (\varphi)$

$\tau_{r-u p}(t)=\frac{1}{2} C_{N}(t) \rho R^{4} \bar{c}_{2}^{2}(S) \hat{r}_{c p}\left(\left(\frac{\Phi n}{2}\right)\left(\frac{d \widehat{\phi}}{d \hat{t}}\right)-\omega_{b}\right)^{2} \cos (\varphi)$

$\tau_{r-\text { down }}(t)=-\frac{1}{2} C_{N}(t) \rho R^{4} \bar{c} \hat{r}_{2}^{2}(S) \hat{r}_{c p}\left(\left(\frac{\Phi n}{2}\right)\left(\frac{d \widehat{\phi}}{d \hat{t}}\right)+\omega_{b}\right)^{2} \cos (\varphi)$

where $\tau_{l-u p}(t)$ denotes the instantaneous torque on the left wing during upstroke, and so on. $C_{N}(t)$ is the instantaneous force coefficient due to delayed stall. $\vec{U}_{b}$ and $\vec{U}_{w}$ are the instantaneous wing velocity vector caused by body angular velocity and wing flapping, respectively, $U_{b}$ and $U_{w}$ denote their magnitudes. $\varphi$ is the rotation angle for simplicity we assume $\varphi$ is symmetric for left and right wings, Therefore,

$$
\begin{aligned}
& \tau_{\text {total-up }}(t)=\tau_{l-u p}(t)+\tau_{r-u p}(t) \\
& =-C_{N}(t) \rho R^{4} \bar{c} \hat{r}_{2}^{2}(S) \hat{r}_{c p} \Phi n \cos (\varphi)\left(\frac{d \widehat{\phi}}{d \hat{t}}\right) \omega_{b} \tau_{\text {total-up }}(t) \\
& \tau_{l-\text { down }}(t)+\tau_{r-\text { down }}(t) \\
& =-C_{N}(t) \rho R^{4} \bar{c} \hat{r}_{2}^{2}(S) \hat{r}_{c p} \Phi n \cos (\varphi)\left(\frac{d \widehat{\phi}}{d \hat{t}}\right) \omega_{b}
\end{aligned}
$$

Thus, the instantaneous total torque could be written in a same form for down and up strokes:

$\tau_{\text {total }}=-C_{N}(t) \rho R^{4} \bar{c} \hat{r}_{2}^{2}(S) \hat{r}_{c p} \Phi n \cos (\varphi)\left(\frac{d \widehat{\phi}}{d \hat{t}}\right) \omega_{b}$

Similarly, we can also write the inertial roll torque by replacing $\cos (\varphi)$ to $\sin (\varphi)$ :

$\tau_{l-u p}(t)=-\frac{1}{2} C_{N}(t) \rho R^{4} \bar{c}_{2}^{2}(S) \hat{r}_{c p}\left(\left(\frac{\Phi n}{2}\right)\left(\frac{d \widehat{\phi}}{d \hat{t}}\right)+\omega_{b}\right)^{2} \sin (\varphi)$

Similarly,

$\tau_{l-\text { down }}(t)=-\frac{1}{2} C_{N}(t) \rho R^{4} \bar{c} \hat{r}_{2}^{2}(S) \hat{r}_{c p}\left(\left(\frac{\Phi n}{2}\right)\left(\frac{d \widehat{\phi}}{d \hat{t}}\right)-\omega_{b}\right)^{2} \sin (\varphi)$

$\tau_{r-u p}(t)=\frac{1}{2} C_{N}(t) \rho R^{4} \bar{c} \hat{r}_{2}^{2}(S) \hat{r}_{c p}\left(\left(\frac{\Phi n}{2}\right)\left(\frac{d \widehat{\phi}}{d \hat{t}}\right)-\omega_{b}\right)^{2} \sin (\varphi)$

$\tau_{r-\text { down }}(t)=\frac{1}{2} C_{N}(t) \rho R^{4} \bar{c}_{2}^{2}(S) \hat{r}_{c p}\left(\left(\frac{\Phi n}{2}\right)\left(\frac{d \widehat{\phi}}{d \hat{t}}\right)+\omega_{b}\right)^{2} \sin (\varphi)$

By summing left and right wing roll torques for each sub-stroke, it can be seen that: 


$$
\begin{aligned}
& \tau_{\text {total-up }}(t)=-C_{N}(t) \rho R^{4} \bar{c} \hat{r}_{2}^{2}(S) \hat{r}_{c p} \Phi n \sin (\varphi)\left(\frac{d \widehat{\phi}}{d \hat{t}}\right) \omega_{b} \\
& \tau_{\text {total-down }}(t)=C_{N}(t) \rho R^{4} \bar{c} \hat{r}_{2}^{2}(S) \hat{r}_{c p} \Phi n \sin (\varphi)\left(\frac{d \widehat{\phi}}{d \hat{t}}\right) \omega_{b} \\
& \bar{\tau}_{\text {total }}=0
\end{aligned}
$$

Thus, the total averaged roll torque over one stroke period is zero as predicted by the simulation results (Fig. 5A).

\section{(C)}

\begin{tabular}{lll}
\hline Symbol & Parameter & Value \\
\hline$R$ & Wing length $(\mathrm{mm})$ & 2.5 \\
$S$ & Wing area $\left(\mathrm{mm}^{2}\right)$ & 2.347 \\
$A_{R}$ & Aspect ratio & 5.96 \\
$\rho_{\text {air }}$ & Air density $\left(\mathrm{kg} \mathrm{mm}^{-3}\right)$ & 1.205 \\
$r_{2}^{2}$ & Second moment of wing inertia & 0.313 \\
$n$ & Flapping frequency $(\mathrm{Hz})$ & 212
\end{tabular}

Table 1. Simulation parameters: Wing area was estimated by regression

\begin{tabular}{|c|c|c|}
\hline Symbol & Parameter & Value \\
\hline$h a$ & Head length(mm) & $0.419 / 2$ \\
\hline $\bar{h} b$ & Head width & $0.813 / 2$ \\
\hline$h \_c$ & Head Height & $0.687 / 2$ \\
\hline$h \_v$ & Head volume $\left(\mathrm{mm}^{3}\right)$ & 0.13 \\
\hline$t_{\bar{a}}$ & Thorax width(mm) & $0.783 / 2$ \\
\hline $\bar{t} b$ & Thorax Height & $0.739 / 2$ \\
\hline $\bar{t} c$ & Thorax length & $0.77 / 2$ \\
\hline$t_{-} v$ & Thorax volume $\left(\mathrm{mm}^{3}\right)$ & 0.24 \\
\hline $\bar{a} \_a$ & Abdomen Height & $1.265 / 2$ \\
\hline$a \_b$ & Abdomen length & $0.773 / 2$ \\
\hline$a \_c$ & Abdomen width & $0.762 / 2$ \\
\hline$a_{-} v$ & Abdomen volume $\left(\mathrm{mm}^{3}\right)$ & 0.41 \\
\hline$\overline{\rho_{\text {body }}}$ & density of mass $\left(\mathrm{mg} \mathrm{mm}^{-3}\right)$ & 1.143 \\
\hline$\theta$ & misalignment of the head w.r.t. the body & $\pi / 6$ \\
\hline $\mathrm{I}_{\text {yaw }}\left(\mathrm{I}_{\mathrm{zz}}\right)$ & Yaw moment of inertia $\left(10^{-12} N \mathrm{~m} \mathrm{~s}^{2}\right)$ & 0.4971 \\
\hline $\mathrm{I}_{\mathrm{roll}}\left(\mathrm{I}_{\mathrm{xx}}\right)$ & Roll moment of inertia & 0.1145 \\
\hline $\mathrm{I}_{\text {pitch }}\left(\mathrm{I}_{\mathrm{yy}}\right)$ & Pitch moment of inertia & 0.5060 \\
\hline
\end{tabular}
equation: $a=-2.023 \times 10^{-6}+1.748 \times 10^{-3} R$, and corresponding aspect ratio is calculated based on equation: $4 R^{2} / S$ [7].

Table 2 Morphology of fruitfly drosophila used in simulation

(D)

The gravitational force and torque of the wing are calculated and subtracted from the measured aerodynamic force and torque; the magnitude of the resultant value $F_{\text {robot }}$ and $\tau_{\text {robot }}$ is then scaled back to the force on the actual fly $F_{f l y}$ and $\tau_{f l y}$ according the equations:

$$
\begin{aligned}
F_{f l y} & =F_{\text {robot }} \frac{\rho_{\text {air }} \cdot n_{\text {fly }}^{2} \cdot S_{\text {fly }} \cdot r_{2}^{2}(s)_{\text {fly }}}{\rho_{\text {oil }} \cdot n_{\text {robot }}^{2} \cdot S_{\text {robot }} \cdot r_{2}^{2}(s)_{\text {robot }}} \\
\tau_{\text {fly }} & =\tau_{\text {robot }} \frac{R_{\text {fly }}}{R_{\text {robot }}}
\end{aligned}
$$

\section{REFERENCES}

[1] M. Sun and J. K. Wang, "Flight stabilization control of a hovering model insect," Journal of Experimental Biology, vol. 210, pp. 2714-2722, Aug 2007.

[2] G. K. Taylor and A. L. R. Thomas, "Dynamic flight stability in the desert locust Schistocerca gregaria," Journal of Experimental Biology, vol. 206, pp. 2803-2829, Aug 2003.
[3] T. S. Collett and M. F. Land, "Visual control of flight behaviour in the hoverflySyritta pipiens L," Journal of Comparative Physiology A: Neuroethology, Sensory, Neural, and Behavioral Physiology, vol. 99, pp. 1-66, 1975.

[4] S. N. Fry, R. Sayaman, and M. H. Dickinson, "The aerodynamics of free-flight maneuvers in Drosophila," Science, vol. 300, pp. 495-498, Apr 2003.

[5] S. P. Sane and M. H. Dickinson, "The control of flight force by a flapping wing: lift and drag production (vol 204, pg 2607, 2001)," Journal of Experimental Biology, vol. 204, pp. 3401-3401, Oct 2001.

[6] S. P. Sane and M. H. Dickinson, "The aerodynamic effects of wing rotation and a revised quasi-steady model of flapping flight," Journal of Experimental Biology, vol. 205, pp. 1087-1096, Apr 2002.

[7] S. N. Fry, R. Sayaman, and M. H. Dickinson, "The aerodynamics of hovering flight in Drosophila," Journal of Experimental Biology, vol. 208, pp. 2303-2318, Jun 2005.

[8] R. Ramamurti and W. C. Sandberg, "A three-dimensional computational study of the aerodynamic mechanisms of insect flight," Journal of Experimental Biology, vol. 205, pp. 1507-1518, May 2002.

[9] T. Hesselberg and F. O. Lehmann, "Turning behaviour depends on frictional damping in the fruit fly Drosophila," Journal of Experimental Biology, vol. 210, pp. 4319-4334, Dec 2007.

[10] M. H. Dickinson, F. O. Lehmann, and S. P. Sane, "Wing rotation and the aerodynamic basis of insect flight," Science, vol. 284, pp. 1954-60, Jun 181999.

[11]S. P. Sane and M. H. Dickinson, "The control of flight force by a flapping wing: lift and drag production," J Exp Biol, vol. 204, pp. 2607-26, Aug 2001.

[12]P. Kodati, J. Hinkle, A. Winn, and D. Xinyan, "Microautonomous Robotic Ostraciiform (MARCO): Hydrodynamics, Design, and Fabrication," Robotics, IEEE Transactions on, vol. 24, pp. 105-117, 2008.

[13]C. P. Ellington, "The Aerodynamics of Hovering Insect Flight. VI. Lift and power requirements," Philosophical Transactions of the Royal Society of London., vol. 305, pp. 145-181, 1984.

[14]S. P. SAME, "THE AERODYNAMICS OF FLAPPING WINGS," in Integrative Biology. vol. Doctor of philosophy: University of California,Berkeley, 2001, p. 107.

[15] R. M.Murray, Z. Li, and S. S. Sastry, A Mathematical Introduction to Robotic Manipulation CRC Press, 1994.

[16] S. Mao and X. Yan, "Dynamic flight stability of a hovering bumblebee," Journal of Experimental Biology, vol. 208, pp. 447-459, Feb 2005.

[17]C. Schilstra and J. H. Van Hateren, "Blowfly flight and optic flow I. Thorax kinematics and flight dynamics," Journal of Experimental Biology, vol. 202, pp. 1481-1490, Jun 1999.

[18]R. Ramamurti and W. C. Sandberg, "A computational investigation of the three-dimensional unsteady aerodynamics of Drosophila hovering and maneuvering," Journal of Experimental Biology, vol. 210, pp. 881-896, Mar 2007. 